EPJ Web of Conferences 66, 02081 (2014)

DOI: $10.1051 /$ epjconf/ 20146602081

(C) Owned by the authors, published by EDP Sciences, 2014

\title{
Microscopic mean field approximation and beyond with the Gogny force
}

\author{
S. Péru ${ }^{1, a}$ and M. Martini ${ }^{2,1}$ \\ ${ }^{1}$ CEA, DAM, DIF, F-91297 Arpajon, France \\ ${ }^{2}$ Institut d'Astronomie et d'Astrophysique, CP-226, Université Libre de Bruxelles, 1050 Brussels, Belgium
}

\begin{abstract}
Fully consistent axially-symmetric-deformed quasiparticle random phase approximation calculations have been performed with the D1S Gogny force. A brief review on the main results obtained in this approach is presented. After a reminder on the method and on the first results concerning giant resonances in deformed $\mathrm{Mg}$ and $\mathrm{Si}$ isotopes, the multipole responses up to octupole in the deformed and heavy nucleus ${ }^{238} \mathrm{U}$ are discussed. In order to analyse soft dipole modes in exotic nuclei, the dipole responses have been studied in $\mathrm{Ne}$ isotopes and in $\mathrm{N}=16$ isotopes, for which results are presented. In these nuclei, the QRPA results on the low lying $2^{+}$states are compared to the 5-Dimensional Collective Hamiltonian ones.
\end{abstract}

\section{Introduction}

One challenge in theoretical nuclear physics is the development of a single approach enabling to describe the excited states of all nuclear systems with the same accuracy. Quasi-particle Random Phase Approximation (QRPA) formalism has been found to be successful in predicting low-lying multipole vibrations as well as giant resonances. These latter provide key informations on nuclear finite-system properties. In the present work, a fully consistent description of resonances in both spherical and axially-deformed nuclei has been undertaken using the QRPA based on Hartree-FockBogolyubov (HFB) states calculated with the Gogny D1S effective force [1, 2]. First, the method and previous studies are presented. Then the results obtained for the heavy and deformed nucleus ${ }^{238} \mathrm{U}$ are reviewed to show the accuracy of the description of low as well as high energy excited states. In order to analyse the mechanisms behind the existence of soft dipole modes in exotic nuclei, theoretical results of the dipole responses in $\mathrm{Ne}$ isotopes and in $\mathrm{N}=16$ isotopes are presented. Finally, in these nuclei, QRPA results on the low-lying $2^{+}$states are compared to the 5-Dimensional Collective Hamiltonian ones.

\section{The method and previous studies}

Within the present method, axially-symmetric-deformed (ASD) HFB calculations are performed in even-even nuclei imposing some symmetries, the time reversal symmetry $T, T \Pi_{2}$ where $\Pi_{2}$ is the

a e-mail: sophie.peru-desenfants@ cea.fr

This is an Open Access article distributed under the terms of the Creative Commons Attribution License 2.0, which permits unrestricted use, distribution, and reproduction in any medium, provided the original work is properly cited. 

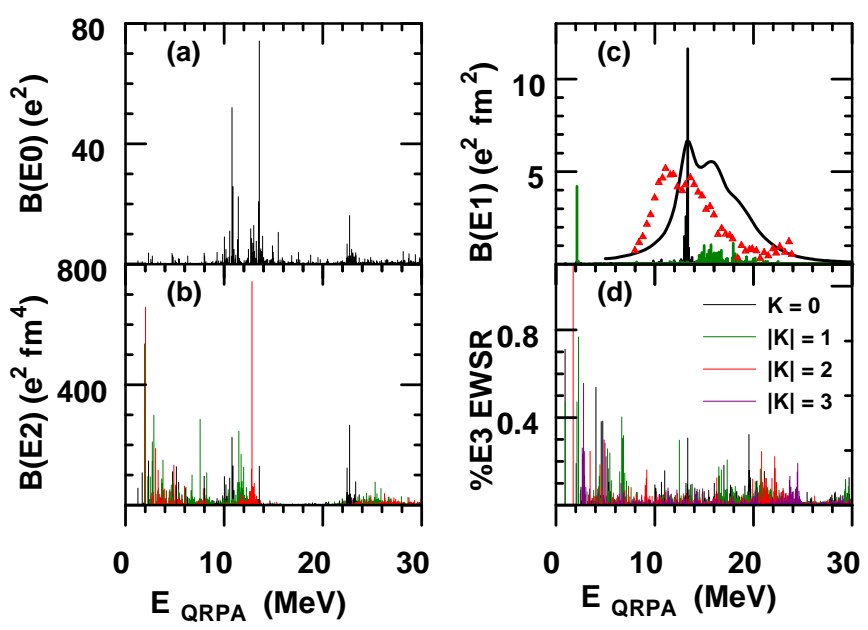

Figure 1. Monopole (a), dipole (c), quadrupole (b) electric transition probabilities and fraction of octupole EWSR (d) in ${ }^{238} \mathrm{U}$ calculated as functions of QRPA excitation energies. In (c) are reported the folding of total dipole strength with a $2 \mathrm{MeV}$-width Lorentzian (solid line) and experimental data (red triangle) from photo-absorption measurements [5].

reflection with respect to the $(\mathrm{xOz})$ plane and also axial and left-right symmetries. Then the projection $K$ of the total angular momentum $J$ on the symmetry axis and the parity $\pi$ are good quantum numbers. Consequently ASD-QRPA calculations can be performed separately in each $K^{\pi}$ block. States $|J M K\rangle_{n}$ of good angular momentum $J$ are obtained projecting the intrinsic excitations, i.e. the ASD-QRPA states $|n K\rangle$. For each multipole, the strength functions are calculated assuming one body operators $\hat{Q}_{\lambda \mu} \equiv r^{\lambda} Y_{\lambda \mu}$, where $Y_{\lambda \mu}$ are the spherical harmonics. In the first work considering this approach with the Gogny force, results on giant resonances have been obtained in ${ }^{22-28} \mathrm{Mg}$ and ${ }^{26-30} \mathrm{Si}$ isotopes [3]. In this study, the role of the ground state intrinsic deformation on strength distributions has been shown. As expected, the dipole response of deformed nuclei was found to be split into two components, one for each $K$ value, and a relation was observed between the sign of the intrinsic quadrupole moment and the $K$ component energy sequence for dipole, quadrupole and octupole responses.

\section{Some recent studies}

More recently, ASD-QRPA calculations have been performed to describe multipolar responses up to the octupole in a deformed and heavy nucleus, namely ${ }^{238} U$ [4]. The multipolar responses are displayed in Fig. 1. Two main peaks characterize the monopole response. The mean excitation energy values of the first and second peaks are 10.7 and $13.4 \mathrm{MeV}$, respectively, in good agreement with values extracted from ${ }^{238} \mathrm{U}\left(\alpha, \alpha^{\prime}\right.$ fission) and ${ }^{238} \mathrm{U}\left(e, e^{\prime}\right.$ fission $)$ reactions studies. The observed splitting is due to the coupling with the $K=0$ quadrupole strength. The peak related to the coupling of the quadrupole mode is the first one, according to the results shown in Fig. 1 (a) and (b). The quadrupole and octupole responses are very fragmented with many collective states predicted below $10 \mathrm{MeV}$. The low quadrupole and octupole state predictions are in agreement with the experimental band heads. Wave functions of low energy QRPA excited states up to $J^{\pi}=8^{+}$have been included in a reaction model to improve the theoretical description of the double-differential cross section for neutron on 

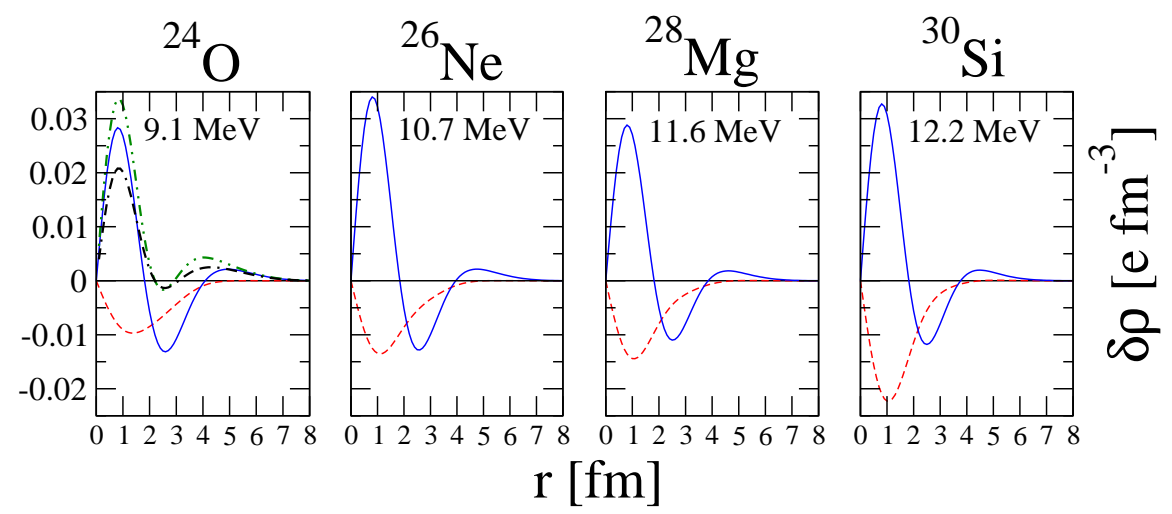

Figure 2. Proton (dashed red line) and neutron (solid blue line) transition densities between low energy dipole state and ground state. For ${ }^{24} \mathrm{O}$ transition densities of neutron single particle-hole states $2 s_{1 / 2} \rightarrow 2 p_{3 / 2}$ (dashed double dotted line) and $2 s_{1 / 2} \rightarrow 2 p_{1 / 2}$ (double dashed dotted line) are drawn to show the collectivity.

uranium [6]. We finally consider the dipole response in ${ }^{238} \mathrm{U}$. It is found to be split into two components: the $K^{\pi}=0^{-}$component is located at an energy lower than the $|K|^{\pi}=1^{-}$one, as expected for a prolate shape. Predictions overestimate by $2 \mathrm{MeV}$ the energy of the dipole response, a value expected to be correlated with particle-vibration coupling (which is neglected here).

The same formalism has been used to study low-energy dipole excitations in neon isotopes and $N=16$ isotones. The results are presented in details in Ref. [7] where the microscopic structure of these low-lying resonances, as well as the behaviour of proton and neutron transition densities are analysed. Concerning the neon isotopes, we found that the strength of the low-energy state increases with the neutron-to-proton ratio. By increasing the number of neutrons the dipole strength distribution becomes more fragmented. A collective low-lying proton excitation is identified in the ${ }^{18} \mathrm{Ne}$ nucleus. Here we report an interesting part of our analysis related to the $N=16$ isotones. For these systems, we obtained excitations between 9 and $12 \mathrm{MeV}$. Figure 2 displays neutron and proton transition densities corresponding to the main low-energy peak. While the giant dipole resonance is always characterized by an isovector behaviour, this low-energy state is more complex. For all $N=16$ isotones, this state is characterized by an isovector behaviour in the center of the nucleus (for $r \leq 2 \mathrm{fm}$ ) and an isoscalar one in the surface region ( $r \simeq 3 \mathrm{fm}$ ). For $r \geq 4 \mathrm{fm}$ one can observe an oscillating finite contribution of the neutrons and a negligible one of the protons. This result does not coincide with the usual isoscalar representation of the pygmy dipole resonance obtained in many theoretical calculations but for heavier nuclei [8]. The spatially extended structure of the $2 s_{1 / 2}$ state is responsible for the shape of the neutron transition density. In order to illustrate this point we have drawn for ${ }^{24} \mathrm{O}$ (first panel of Fig. 2) the unperturbed transition densities for the two dominant excitations $v 2 \mathrm{~s}_{1 / 2} \rightarrow 2 \mathrm{p}_{3 / 2}$ at $8.92 \mathrm{MeV}$ and $v$ $2 \mathrm{~s}_{1 / 2} \rightarrow 2 \mathrm{p}_{1 / 2}$ at $10.33 \mathrm{MeV}$. It clearly appears that unperturbed and QRPA transition densities are quite similar at the center of the nucleus, reflecting the crucial role of the $v 2 \mathrm{~s}_{1 / 2}$ state, while they differ in the surface region, showing the importance of correlations. In addition to the dominant transition other configurations contribute to generate the low-lying resonance, as quantitatively analysed in Ref. [7]. This small but finite collective behaviour characterizes all the $N=16$ analysed isotones.

To investigate further the QRPA predictive power, we now compare our QRPA results with the ones obtained in the 5DCH framework [9, 10]. In Fig. 3, QRPA energies and transition probabilities of the first $2^{+}$excited state are compared with experimental data and $5 \mathrm{DCH}$ predictions for $\mathrm{N}=16$ 

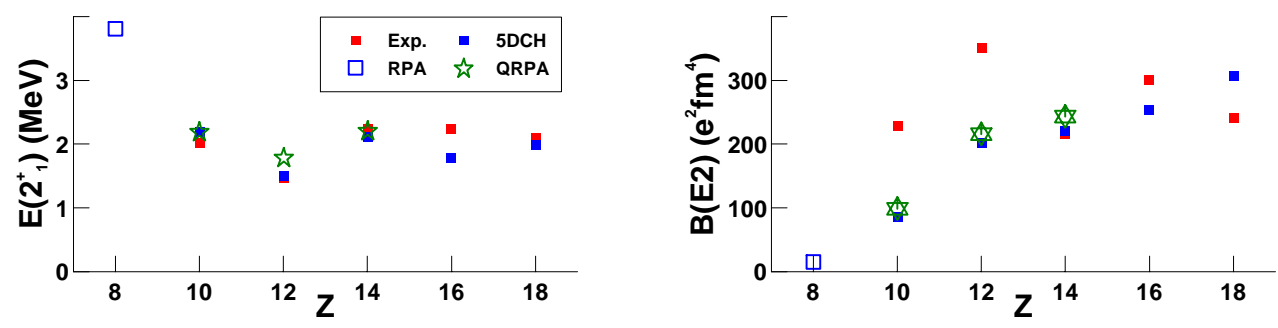

Figure 3. Energies and transition probabilities of the first $2^{+}$excited state of $\mathrm{N}=16$ isotones calculated with different methods and compared with experimental data.

isotones [11]. These values show that both approaches are able to provide structure informations in harmonic nuclei with quite the same accuracy, especially for $\mathrm{N}=16$ isotones. Nevertheless the 5DCH approach is not able to describe nuclei which are too rigid against the deformation and overestimates the transition probabilities while the QRPA approach is not able to take into account secondary minima.

\section{Summary}

The QRPA approach adapted to axially-symmetric-deformed nuclei has been applied to light exotic nuclei and to the heavy and deformed nucleus ${ }^{208} \mathrm{U}$. Results on dipole mode are in good agreement with experimental data. For spherical nuclei, the QRPA formalism completes the 5DCH predictions including the same effective interaction.

\section{References}

[1] J. Dechargé and D. Gogny, Phys. Rev. C 21, 1568 (1980).

[2] J.F. Berger, M. Girod, and D. Gogny, Comp. Phys. Comm. 63, 365 (1991).

[3] S. Péru, H. Goutte, Phys. Rev. C 77, 044313, (2008).

[4] S. Péru, G. Gosselin, M. Martini, M. Dupuis, S. Hilaire and J.-C. Devaux, Phys. Rev. C 83, 014314 (2011).

[5] G. M. Gurevich et al, Nucl. Phys. A273, 326 (1976).

[6] M. Dupuis, S. Péru, E. Bauge and T. Kawano, proceedings of the 13th International Conference on Nuclear Reaction Mechanisms, Varenna 2012, CERN-Proceeding - 002, p95 (2012).

[7] M. Martini, S. Péru and M. Dupuis, Phys. Rev. C 83, 034309 (2011).

[8] N. Paar, D. Vretenar, E. Kahn, and G. Colò, Rep. Prog. Phys. 70, 691 (2007).

[9] G.F. Bertsch, M. Girod, S. Hilaire, J.-P. Delaroche, H. Goutte, and S. Péru, Phys. Rev. Lett. 99, 032502 (2007).

[10] J.-P. Delaroche, M. Girod, J. Libert, S. Hilaire, H. Goutte, S. Péru, N. Pillet, and G.F. Bertsch, Phys. Rev. C 81, 014303 (2010).

[11] A. Obertelli, S. Péru, J.-P. Delaroche, A. Gillibert, M. Girod, and H. Goutte, Phys. Rev. C 71, 024304 (2005). 